\title{
CAUSAL NETS
}

or

What is a Deterministic Computation?

\author{
Péter Gacs 1 \\ University of Rochester \\ Leonid A. Levin 2 \\ Massachusetts Institute of Technology
}

TR 88

March 1981

ABSTRACT. We introduce the concept of causal nets -- it can be considered as the most general and elementary concept of the history of a deterministic computation (sequential or parallel). Causality and locality are distinguished as the only important properties of nets representing such records. Different types of complexities of computations correspond to different geometrical characteristics of the corresponding causal nets -- which have the advantage of being finite objects. Synchrony becomes a relative notion. Nets can have symmetries; therefore it will make sense to ask what can be computed from a rbitrary symmetric inputs. Here, we obtain a complete grouptheoretical characterization of the kind of symmetries that can be allowed in parallel computations.

1Author's address: Computer Science Department, University of Rochester, Rochester, New York 14627. Part of this work was done while this author was visiting Johann Wolfgang Goethe University, Frankfurt am Main in 1978 and Stanford University in 1979.

2Author's address: Laboratory for Computer Science, Massachusetts Institute of Technology, Cambridge, Massachusetts 02139 . The research of this author was partially supported by NSF Grant MCS 77-19754. 


\section{Introduction}

In this work, we propose a framework unifying various aspect,s of the theory of complezities of information processing-also proyiding a language for some new problems. Presently, many results belo: the level of abstraction provided by Blum's computational complexiby heory are seemingly dependent on speciac machire nodels (Turing mentin, RAM, iterative netwo: $k$, etc.) or formulated in such models with suine commeni on the measure of independence of the model. This leads to unnecessary specification and to aw $k$ ward formal constructions unusual in traditional mathematics.

We take the notion of computation itself as a primitive (causal net ${ }^{1}$ ) instead of considering the work of a device performing this computation. Such an approach is less detailed since the same computation can be implemented in various ways: on different devices, sequentially of parallelly, varying the order of the operations and their distribution over parts of the device. Due to its potential for the avoidance of details, we bope to set up a more unifled framework providing simpler definitions and still preserving concreteness and elementarity. A causal net can be interpreted as the time-space history of all elementary operations accomplished in the computing process, with their mutual dependencies indicated. As an additional advantage of this approach, a computation on each input is regarded as a separate finite object independently of the context of a function over an infinite domain. In this way, we hope to facilitate the application of geometric and algebraic methods in complexity theory, and to preserve the advantages of the theory of Boolean networks.

Unlike other types of nets (e.g. Boolean networks) the causal net constructs its logical structura in the process of computation and inus it can be reconstructed from its input and the structure of the possible neighborhoods in it (causal structure). All operations needed for this are taken into account. At a fixed causal structure (playing the role of the program of the algorithm) the in put nets can ba arbitrarily large. At a given size of the input, the size of the causal nets is a complexity of computation in the usual sense (most similar to the product of time and space) in contrast to the size of the Boolean networks which is bounded (by $2^{n} / n$ ). The closeness of the definition of causal nets to some physical ideas gives hope to find a connection between the geometrical characteristics of these nets and the physical characteristics of computations, as e.g. the size of the net and the entropy increase caused by the computation in question.

1 N. V. Petri in [2] is different iroin the inventor of Petri nets- which have no essential relation to our causal nets. 
The last years witnessed a large number of ad hoc models for parallel computation addressing special problems like synchrony. Some of them, as also the classical Boolean networks, are very different in nature from Turing machine-style sequential nodels. For secuentisl machines, Kolmogorov and Uspenskii [1] made the first significant sieps toward a model general enough so that most other models could be considered as its restricted forms. Their machine has a graph-like storage sbucture undergoing gradua! local changes in time, by the work of a consingt number of active units.

In the next sections, we introduce the concept of causal nets and compare it with a mor traditional model of computations: Kolmogorov machines in parallel mode. We also consider the problem of computability when input nets with arbitrary symmetries are allowed. This problem seems to be new because it does not arise but for sufficiently general concepts of parallel computations like the ones presented here. We give a complete characterization of the functions computable in these models -in terms of the automorphism group of the input. The result can be considered as some "Church Thesis" for symmetry-preserving computations and is related to some combinatorial theorems of Babai and Lovász [5]. L. A. Levin originated the concept of causal nets, P.Gács proved the result on the symmetric inputs.

\section{Basic definitions}

Causal rets

A net $X$ is a directed labelled graph, i.e. a matrix $\theta:|X|^{2} \mapsto \theta$ defining the label $\theta(x, y)$ of the edge (or the symbol $\infty$ of its absence) between any two nodes. A subnet is the restriction of $\theta$ to a subset of the nodes. The input subnet is the union of all oriented cycles. The cause $\lfloor x\rfloor$ of a node $x$ is the subnet of nodes $y$ for which $(y, x)$ is an edge. The immediate consequence $A^{+}$of a subnet $A$ is the subnet containing $A$ and all nodes the entire cause of which is contained in $A$.

A net represents the whole space-time history of a computation rather than its state at some time moment. A node of the net corresponds to an "elernentary event" in the course of the computation, the edges to "causal relations" between them. We can (and will) use multiple edgessimulated by adjusting the set $\theta$, and states for the nodes-simulated by the states of the preceding edges. 
Definition 1 A net is called local if the cause of each node is weakly connected (i.e. connected as an undirected graph). A local net is called causol if any isomorphism between its two subnets $A$ and $B$ can be uniquely extended to an isomorphism between $A^{+}$and $B^{+}$.

The requivenerit, of uniqueness is not essential and is inposed only for convenience. To check for causality and locality, only sulmets isomorphic to causes of nodes should be considered. This is easy sirce all such subnets are small and connected.

The requirement of causality is the way we represent physical determinism: the past uniquely determines the future. An other important physical principle, that of the locality of interaction requires that the immediate cause of an elementary event should consist of events closely related to each other. The evidence of this close relation is usually present in a chain connecting these events and should be considered as part of the cause. Thus, nodes of the cause of a node have causal interconnection themselves and therefore correspond to close but different momenis of time (in some analogy to the formalism in mechanics where the future positions of a system are determined by its present position and a position in the near past-giving a speed).

The noninput nodes and the strongly connected components (packets) of the input form an acyclic graph with a natural partial order $\leq$ on it. The base subnet consists of the input and all preceding nodes. The output subnet consists of the noninput nodes adjacent to edges labelled by a distinguished output subalphabet $\theta_{O}$. Any graph can be converted to an input net by adding a loop to each node. These are the usual bases for nets. Other types of base may be used to simulate fancy things, e.g. the use of "oracles" (input nodes whose cause contains noninput nodes).

The nodes of a net can be objects of any kind. But a noninput node $x$ can be naturally identified with the function mapping $y \in\lfloor x\rfloor$ to $\theta(x, y)$. In the case of a single-label alphabet, $x$ can be identified with $\lfloor x\rfloor$. Then the causality requirement for a net $X$ can be written as $|X| \supseteq x \cong y \in$ $|X| \Rightarrow x \in|X|$.

\section{Programming}

A causa! net can in general be described much shorter than by listing the entire matrix $\theta$. It is already uniquely deterinined by its base and the types of neighborhoods that can occur in it (unlike the Boolean networks). The neighborhood $V(x)$ of a node $x$ (its center) is the subnet consisting of $x$ and all nodes connected to $x$. The causal neighborhood $C(x)$ contains 
$x$ and $\lfloor x\rfloor$. The local [caisal] structure (program) of a net is the set of its [causal] neighborhoods or "commands" (up to isomorphism). A net $X$ is said to be consistent with any local [causal] structure containing the one of $X$.

A (local or causal) program $P$ is said to generate a net, $X$ if $P$ is consistent with $X$ but with no extonsion of $X$ having the same input. The minimal progran generating $X$ is the program of $X$. Tivery causal program generatos a uniquely determinad (possibly infinite) not from any base and any net is generated from its base by its program. If the net is finite and the output exists in all connected components, we say that the output is computed from the base by the program. Thus to implement computations by these concepts, take a finite causal program $P$ and input $A$, let the program start generating a causal net from it by subsequent extensions and take the output as the result.

The requirement of consistency with some fixed local structure is a useful way to impose various local restrictions on the net, e.g. boundedness of the degree of the nodes. The computation by a causal net is monotone: from a part of the input, always a part of the output will be computed. To eliminate this effect, one can always confine oneself to functions in whose domain no input net is a proper part of an other one. Such a domain is, for example, the set of all nets consistent with a closed local structure as defined below. Also, in a closed net, we can easily recognize the last moment when a node was used in generating other nodes.

Definition 2 A net is locally asymmetric [closed] if none of its neighborhoods has a nontrivial isomorphism to itself [to a proper part of another one]. A closed locally asymmetric net with one distinguished centra! node in each (reakly) connected component is called marked.

The nodes of a connected marked net can easily be numbered in a canonical way: we construct a spanning tree with the central node as the root, proceeding on the edges of $X$ from the root e.g. in a breadth-first manner. In the theory of information processing, we pracically never encounter nonmarked nets, and the permission of symmetric nets gives rise to serious special problems (like the problem to find an algorithm deciding whether two given graphs are isomorphic).

Example: representation of a Turing machine

A Turing machine has a tape-a finite succession of cells numbered 
by subsequent integers, and a head observing the cell with number $c(t)$ at time $t$. A finite set of states is fixed and each cell $k$ as well as the head is at each moment $t$ in one of these states $p(t, k)$ and $q_{t}$. The terminal cells have the distinguished states $R$ and $L$. The program of the machine is a finite function $\lambda$ ordering certain actions to pairs of stsces. Thus, $\lambda\left(q_{t}, p(t, c(t))\right)$ determines $q_{t+1}, p(t+1, c(t)), c(t+1)-c(t)= \pm 1$ and $p(t+1, k)=p(t, k)$ for all $k \neq c(t)$. If the cell $c(t+1)$ does not exist yet, it will ba created. If the head was at one of the ends it also determines whether the cell $c(t)$ has to be removed. The sequence $p(0, k)$ is the input and $c(0)=0$. Thus always $c(t) \equiv t(\bmod 2)$, and since the state of a cell cannot change in steps of different parity, we can exclude these from consideration. Let us agree that at the end of the computation, the head assumes a special state $\tau$, and going from one end of the tape to the other one, erases it. (This prevents the representing causal net from being infinite.)

To represent the computations of this machine by causal nets, let $s(t, k)$ denote $(p(t, k), x)$ where $x$ is $q_{t}$ if $c(t)=k$, special symbol otherwise. Let the set $V$ of nodes of the causal net be the set of time-cell pairs $(t, k)$ of equal parity where the cell $k$ exists at time $t$. The edges run between nodes $(t, k \pm 1)$ and $(t+1, k)$. Their label reflects the states $s(t, k)$ of their adjacent nodes. Other edges, with some constant label, run between $(t-1, k)$ and $(t+1, k)$. If the cell $k$ does not exist at moment $t-1$, this edge connects $(t+1, k)$ to the terminal cell or forms a loop when $t+1 \leq 1$. The output subalphabet contains the labels with states $s(t, k)$ having $x=\tau$.

It can be easily checked that the above defined net is causal and local.

\section{Complexity of computations}

\section{Time and space}

One of the differences between the more traditional models and the computations as modelled by the causal nets is that on the latter the elementary operations are not necessarily synchronized. Only the relative order of those events is determined which are in a causal relation to each other. What results is a certain vagueness in the definition of the storage requirement of a causal net.

Let us define the height $d(x)$ of a node $x$ of a causal net as the maximum length of a decreasing sequence of nodes starting with $x$. The height 
of a whole connected net $X$ is $D(X)=\max _{x \in V} d(x)$. The height can be considered as the time required for the computation. Let $\Phi(x)$ be a monatone mapping of $|X|$ to the axis of time. (An example is $d(X)$.)

Definirion 3 The storage size $s,(t, X)$ at moment $t$ the number of edges $(x, y)$ with $\Phi(x) \leq t$ and $\Phi(y) \geq t$. Denote $s_{\phi}(X)=$ $\max _{t} s_{s}(t, X)$. For an unconnected not, height and storage are defined componentyise, as a family of nwmbers indexed by the connected components of $X$.

It seems to be unnatural to define the storage used at one moment in a way independent from the time function $\Phi(x)$; apparently by the same considerations that in the theory of relativity show that there is no invariant way to define the notion of two events occurring at the same time. (Note that any imaginable relativistic computer is representable by a causal net.)

Minimizing the storage size over all possible monotone mappings we obtain the value $s_{0}=\min _{\Phi} s_{\Phi}(X)$ that is similar to the number of stones needed to "pebble" the net (see [6]). However, $s_{0}$ is not a realistic measure of storage requirement. It seems to be reasonable to require that a timing be realized by the height function of some net "implementing" $X$ in some formal sense. And the minimizing timing may be hard to compute and not implementable.

\section{Time-space trade-off}

Machines that actually build up a causal net of size $n$ from its program and input cannot require less storage than $n$. The situation changes ii we are content with a machine that does not necessarily store a representation of the net, only gives $\theta(i, j)$ for any two nodes (their numbers) $i, j$ on request. (The machine weakly represents the net.) This may require only storage $O(\log n)$ instead of $n$ (that never requires more is another formulation of the hypothesis of logarithmic time-space tradeoff). The next theorem was originally proved by N. V. Petri [2] in terms of some concrete types of machines, but causal nets are the most natural setting for formulating it. It says that the storage size for weak representation can be minimized (no speedups).

Theorem 1 For any causal structure $P$, there is a Turing machine $T$ with the following property. For each input net $X$, using a weak representation of $X$ (by an oracle), is weahly represents a causal net $Y$ generated by $P$ from $X$. Any other Turing machine $M$ doing this (even only) for $X$ will 
use storage no less than by a constant $C_{M}$ times the storage used by $T$.

Sketch of proof: The optimal Turing machine $T$ works as follows. It sets forth a certain amount of storage $s$, then considers all possible other Turing machines $M$ with a description shorter than $s$. Ruining over all $k$-tuples of numbers less than $2^{s}$, where $k$ is the maximum size of the elements of the causal structure $P, T$ is able to decide whether $M$ while working within storage $s$ weakly regresonts a net genereted from $X$ by $P$. If it does not find any $M$ doing that, it increases $s$. When it finally finds a fititiag $M$, it uses $M$ for answering the questions it was asked.

\section{Example: Characterization of Pointer Machine complexity}

Various models of computation with only one finitary operation at each step can be considered as essentially a special case of Kolmogorov's graph machine [1]. This differs from the "storage modification machine" proposed later by Schönhage [3] and called "Pointer Machine" by Knuth only in that Schönhage works with directed, Kolmogorov and Uspenskii with undirected graphs (forcing thereby both bounded in- and outdegree). The storage structure, called pointer graph of the Pointer Machine is a directed labelled graph with constant outdegree.

The program prescribes how the central node transforms its 2-neighborhood step-by-step, modifying thereby gradually the whole graph. The initial graph is the input, the graph at halting is the output. They are labelled by the disjoint alphabets $\theta_{I}, \theta_{O}$.

Barzdin' and Kalnin's generalized the model of Kolmogorov and Schönhage by introducing parallelism. A program for tha Parallel Pointer Machine (PPM) will be similar to the program of a PM but its meaning is different: the local transformations must be simultaneousiy carried out by all nodes. A node $x$ changes only its outgoing edges, or disappears if they all loop. A common new node may be created by a maximal clique formed by edges with a distinguished label $\epsilon$. In determining the next action, edges with output labels do not count. The computation is finished when all edges have output labels. A PPM is a parallel Kolmogorov machine (PKM) if its pointer graphs are undirected at each step (i.e. their matrix is symmetric and each node has a loop with a special constant label). The set of nonempiy undirected pointer graphs is denoted by $T(\theta)$.

The functions over undirected connected marked graphs computable by the PM and PPM are exactly the recursive functions. With respect, to computing time, the PPM is a poweriul generalization of the PM and is 
able to solve e.g. any NP problem in polynomial time (but possibly with exponertial space). This model can claim ability to simulate any other model of parallel computation efficiently.

A funcion $f$ computable by a PPM-just as the coinplexities in Definition $3-i s$ componentwise, i.e. commutes with discomacted union: $f(X \cup Y)=f(X) \cup f(Y)$ if $|X| \cap|Y|=0$. We associata a pointer graph $Z$ with a (pos3ibly acyclic) net $Z$ by identifying all nodes connected by edges with a special label $\eta$.

Note that the above version of the PPM is more general than usual in ordor to extend Theorem 2 to symmetric inputs. For usual computations, the inputs should be assumed marked.

Theorem 2 For componentwise functions $f, u, v$ over $\mathrm{T}\left(\theta_{l}\right)$ these properties are equivalent:

(a) A PKM exists computing $f(X)$ for each $X$ in time $O(u(X))$ and storage $O(v(X))$.

(b) For each $X$ a closed causal net $Y$ exists with bounded degrees of nodes, with input $X$, output $Z$ with $Z^{\prime}=f(X), D(Y)=O(u(X))$, $s_{d}(Y)=O(v(X))$.

The proof will be given in the Appendix.

OPEN PROBLEM Find out which traditional complexity corresponds to the size of causal nets. It is known that the size of the smallest. causal net computing a function is between the time required on a PM and the time required on an "address-machine" (a PM with a tree-like storage structure). The second complexity may exceed the first one only by a logarithmic factor. 


\section{Synmetric inputs}

In this section, we will characterige the functions computeble by causal nets. Of course, every such function is jatial recursive. But it turns out that partial recursive functions that se deined on certain zery symmetric inputs are not computable in modats oreserving this symatiny.

Let us try, e.g., to compute $n$ (wot 2 ) from a "circle $X$ of length $n$ ": some net with the automorphism gro:j $Z_{n}$ (the cyclic group of order $n$ ). We ask for a program generating a ons-adge output $z$ from $X$ with state equal to $n(\bmod 2)$. Thinking in terns of parallel pointer machines, we can imarine the input as a circular array of identical automata-capable of unlimited local organization and creation-trying to merge into a single node. There is no leader among them to organize the process. Since all have similar initial neighborhood, the first merge can divide them only into small groups of identical size-which is impossible if their number is prime. Indeed, it turns out that the existence of such a program implias that $n$ cannot have any large prime divisors. (Such numbers are sometimes called "smooth", in reference to smooth sand containing only fine grains.)

The functions computable on the Pointer Machine are exactly the partial recursive functions. However, the input to a PM must alyays be a marked pointer graph. Theorem 2 sets up a correspondence between functions computable by causal nets and those computable by the Parallel Pointer Machine. Hence if we restrict our input nets to be marked, the functions computable by causal nets are just the partial recursive functions. On the other hand, functions that are not computable by causal nets will therefore be not computable by the Parallel Pointer Machine (a version of Theorem 2 holds also without ine rastriction that the PPMI be a Kolmogorov machine). We now proceed to formulate the necessary and sufficient condition s for a recursive function without the markedness requirement to be computable by a causal net.

A partial componentwise function $f$ from nets with a loop-edge at each node to output nets with uniformly bounded indegree will be called standard.

Let $P$ be a causal structure generating causal nets $X_{0}, X_{1}$ with outputs $B_{0}, B_{1}$ from input nets $A_{0}, A_{1}$. Suppose further that there exists an embedding $\iota$ of $A_{0}$ into $A_{1}$. By causality, this embedding will generate an embedding of the whole causal net $X_{0}$ into $X_{1}$ and thereby an embedding $\iota^{p}$ of $B_{0}$ into $B_{1}$. Notice that the image of $B_{0}$ will be an ideal $C$ of $B_{1}$ $(y<x \in|C|$ implies $y \in|C|)$. For different causal structures $P$ compnting $f$ this correspondence of embeddings on the outputs to embeddings 
on the inputs can be different, but its existence is a serious restriction implying among others the monotonicity of $f$. Hence the first condition on the standard partial function $f$ is the following. Let id $A, B$ be the identical embedding of $A \subseteq B$ into $B$.

(i) There exists a recursive correspondence $F$ which ciders an isomotphism $\iota^{F}$ of $f\left(A_{0}\right)$ onto an ides of $f\left(A_{1}\right)$ to each enveriaing $\iota: A_{0} \mapsto$ $A_{1}$. F is a functor, i.e. $\left(\iota_{0} \circ \iota_{1}\right)^{f}==\iota_{0}^{F} \circ \iota_{1}^{F}$. Let $A_{0}, A$ be subnets of net $C, A_{2}=A_{0} \cap A_{1}, B_{j}=\mathrm{id} A_{j}, C\left(f\left(A_{j}\right)\right)$. Then $B_{2}=B_{0} \cap B_{1}$.

This intersection property of the functor $F$ reflects the icet that the net $B_{2}$ computad by a program $P$ from the intersection $A_{2}$ of two nets $A_{0}, A_{1}$ is the intersection of the nets $B_{0}, B_{1}$ computed from $A_{0}$ and $A_{1}$ respectively. Indeed, $B_{2} \subseteq B_{0} \cap B_{1}$ is evident from monotonicity. But the ancestors in the input of each node of $B_{0} \cap B_{1}$ are all both in $A_{0}$ and $A_{1}$, hence also in $A_{2}$. This proves $B_{2}=B_{0} \cap B_{1}$.

The above property implies that for a subnet $B$ of an output net $f(A)$ we can find the smallest part of $A$ still producing $B$. For any subnet $A_{0}$ of $A$ define $f\left(A_{0} ; A\right)=\mathrm{id}_{A_{0}, A}^{F}\left(f\left(A_{0}\right)\right)$; this is the subnet of $f(A)$ computed from the subnet $A_{0}$ of $A$. The set of ancestors $f^{-1}(B ; A)$ of $B$ is the intersection of all subsets $A_{0}$ of $A$ with $f\left(A_{0} ; A\right) \supseteq B$. (Notice that this notion is defined only by the functor $F$, without causal nets.) It follows from (i) that $f\left(f^{-1}(B ; A) ; A\right) \supseteq B$. In a causal net $X$, of course, a node $a$ of the input $A$ is the ancestor of a subset $C \subseteq|X|$ if $a \leq y$ for some $y \in C$. Notice that since the image of $\iota^{F}$ is always an ideal, $a<b$ implies $f^{-1}(\{a\} ; A) \subseteq f^{-1}(\{b\} ; A)$.

(ii) For each input $A$, the set of ancestors of each node of $f(A)$ is connected.

The most interesting property $F$ must have is connected with possible symmetries of the inputs. The functor $\lambda \mapsto \lambda^{F}$ is a homomorphisra from the group of automorphisms of $A$ to that of $f(A)$. For any node $x$ of $f(A)$, let us denote by $G(x, A, F)$ the factorgroup of the group of all automorphisms $\lambda$ that leave $x$ invariant (i.e. for which $\lambda^{F}(x)=x$ ) by the normal subgroup of the autornorphisms that fix all elements of $f^{-1}(\{x\} ; A)$. (This divisor is the unity if $x$ depends on the whole input.)

For any finite group $G$, let $a(G)$ be the minimum of the indices of proper subgroups in $G, b(G)$ the maximum of $a(H)$ over all subgroups of G. $b(G)$ is sometimes called the smoothness of $G$ in analogy to the abovementioned notion of smoothness of natural numbers.

(iii) $b(G(x, A, F))$ is bounded for all inputs $A$.

Theorem 3 For a standard partial function $f$, the following two condi- 
tions are equivalent.

(a) For all nets $A$ in the domain of $f$, there are finite causal nets with input $A$, output $f(A)$, and with bounted indegrees of noningal; nodes.

(b) $f$ satish is: (i-iii).

The poof will be given in the Appendix.

Fentaks

1. The recursiveness of the functor in (i) cannot be replaces by the weaker requirenent of the recursiveness of the function $f$. In the $\Lambda$ ppendix, we give an example of a function $f$ with a nonrecursive functor satisfying the rest of (i-iii) which has no recursive functor (even without the rest of (i-iii)).

2. Of most interest are functions in whose domain no net is a proper part of an other one, and which are invariant, i.e. their functor $F$ maps any automorphism of the input into the identity on the output. In this case, (iii) requires the automorphism groups of inputs to be uniformly smooth.

3. The smooth groups play an important role in the newly discovered isomorphism-testing algorithms of graphs of bounded valence [7]. We plan to follow up the consequences of [7] in a following work. Notice also that the automorphism group of a connected graph of bounded valence is smooth if one of its orbits is small.

\section{Conclusion}

Causal nets might become a simple and universal concept in the theory of computation: they provide an easy and natural way to describe the work of different real and inaginary computing devices since nothing occurs in their definition but the most general physical ideas concerning the processes going on in the machines. The causal net can be constructed already on the basis of the computation to be accomplished without specifying the type of machine used. Different characteristics of the computing resources correspond to simple geometric characteristics of the causal nets. Besides their universality, the causal nets have the advantage of a sinple definition and give the possibility to consider each computation as a singia finite object independently from the context of all possible 
computations of the same algorithm on different inputs. 'This makes common geornetrical and algebraic methods available for the study of computations. At the same time, the theory of causal (in contrast to the Boolean) nois is equivalent to the theory of algorithms $y$ ia the fact that a net is uniçuely reconstructable rom its ingut net ir is ausal structure is known.

\section{Appendix}

\section{Proof of Theorem 2}

The computation of the PPM is a series of pointer graphs $X=$ $X_{1}, \ldots, X_{u}=f(X)$. Let $p_{t}(x, y)$ be the label of the edge $(x, y)$ in $X_{t}$ ( $\infty$ if this edge does not exist). We construct a causal net $A$ oyer the nodes $(t, x)$ for all $x \in X_{t}$ having at time $t-1$ nonoutput outgoing edges. Put $\theta\left((t, x),\left(t^{\prime}, y\right)\right)=p_{t}(y, x)$ for all $x, y$ in $X_{t}$ where $t^{\prime}=t+1$ or $t^{\prime}=$ $t=0$. Connect also, by edges having some new constant label, all pairs $(s, y),(t, x)$ where $y$ is at time $t-2$ in the 2-neighborhood of $x$-or of a node that created $x$ if $x$ is new-and $s=t-1, t-2$. If $x$ has an ouiggoing output edge, connect $(x, t)$ and $(x, t+1)$ with an $\eta$-edge. $A$ can be seen to be causal and closed. Its input graph is $X$. Its output graph $Z$ contains a path of $\eta$-edges for each node of $f(X)$. After the contraction of these paths, we get $Z^{\prime}=f(X)$.

It remains to prove $(b) \Rightarrow(a)$. For a closed local structure $\mathcal{Q}$, let us call its $c$-domain the set of all input graphs $X$ frorn which $\mathcal{Q}$ computes a net $Y$ with output $Z$ satisfying $Z^{\prime}=f(X), D(Y) \leq c u(Y), s_{d}(Y) \leq c v(Y)$. By the assumption of the theorem, any $X$ is in the domain of some $\mathcal{Q}$ with degrees of nodes bounded by some number $k$. Notice also that if $X_{1} \cup X_{2}$ is in the domain of $\mathcal{Q}$ then so is $X_{1}$. It follows that a local structure $\mathcal{Q}$ exists whose domain is the set of all nets.

We can therefore suppose that $Y(X)$ is generated from $X$ by $\mathcal{Q}$ applying subsequent extensions. We have to show that $Y$ can be built up by a PPM (within the required time and storage bounds) level-by-level. In this construction, we will first use some temporary output labels $\bar{\alpha}$ when some edge of the net should occur with output label $\alpha$. $\eta$-edges will be contracted as soon as possible.

Let $A_{t}$ be the subnet of nodes of height $\leq t$ in $Y$. Let us omit from $A_{t}$ all noncutput nodes which are closed: whose neighborhood is isomorphic to an element of $\mathcal{Q}$. (These nodes cannot occur in the cause of any new 
node, so they are no more needed). The resulting subnet is $B_{t}$. The program for the PPM coinputes $B_{t+1}$ from $B_{t}$ in a constant number of steps in the following stegss.

1. For all nodes $x$, the mache looks up all copies $U$ of the cause of some command $Z$ of $Q$ continiag $x$. This needs only constantly many sieps since the the degree $v$ ? the nodes of $B_{t}$ is bounded by $k$. For each such $U$ and $Z$, a new inliary node $v(x, Z, U)$ is added, with pointers having the same velusis as the pointers at 2 .

2. Each $v(x, Z, U)$ and $v(Z, Z, U)$ is connected by a pointer with label $\epsilon$ in both directions forming thereby $\epsilon$-cliques $M(Z, U)$ for the third stage.

3. The $\epsilon$-cliques are replaced by single nodes with the corresponding pointers.

4. If a node $x$ is closed do the following. If $x$ is not an output node and has no adjacent $\eta$-edge then delete it. If $x$ is connected to an other closed node by an $\eta$-edge then merge them. Thereafter, if $x$ is an output node, convert all temporary output edges leaving $x$ to the corresponding final ones (which are also output edges of the KPM we are just defining).

\section{Proof of Theorem 3}

1. To prove that (a) implies (b) it is enough to show that (a) implies (iii): the rest has been shown already. Let $Y$ be any command of a causal structure $P$. It can have automorphisms of its $o w n$, which divide the cause of $Y$ into orbits (transitivity classes). Denote by $h(P)$ the maximum size of orbits in all commands in the program $P$.

Suppose that (a) holds, i.e. that some causal structure $P$ generates from every input net $A$ a causal net $X$ with output $B=f(A)$. Let $z$ be a node of $B$. Let $F$ be the functor naturally provided by the net. We will show that $b(G(x, A, F)) \leq k(P)$.

Let $C$ be any subset of $X$. Let $G(C)$ be the group of automorphisms of $A$ leaving each node of $C$ fixed. Let $C^{0}$ be the set of ancestors of $C$ in $A$. We will show that $b\left(G(C) / G\left(C^{0}\right)\right) \leq k$. We will use induction over the following partial ordering $\prec$ of sets of nodes of $X . C_{1} \prec C_{2}$ if $C_{1} \supseteq C_{2}$ and every element $x$ of $C_{1}$ is majorized by an element $y \geq x$ of $C_{2}$. A set $C$ is minimal in this order only if it contains $C^{0}$. In this case, $G(C)$ is the ona-element group. Suppose that the assertion is true for all $C^{\prime} \prec C$. If the cause of every node of $C$ is in $C^{\prime}$ then $C$ contiains $C^{0}$. Suppose 
that $C$ contains a node $x$ for which $\lfloor x\rfloor \& C$. Let $y \in\lfloor x\rfloor-C$. Put $C^{\prime}=C \cup\{y\}$. By our inductive assumption, $b\left(G\left(C^{\prime}\right)\right) \leq k$, since $C^{\prime} \prec C$. We show that $\left|G(C): G\left(C^{\prime}\right)\right| \leq k . G\left(C^{\prime}\right)$ consisis of all elements of $G(C)$ that leave $y$ fixed. To each coset of $G\left(C^{\prime}\right)$ in $G(C)$, a different node of $\lfloor x\rfloor$ will correspond which is, moreover, in the oubit of $y$. Therefore the number $\left|G(C): G\left(C^{\prime}\right)\right|$ of cosets is bounded by the maximum of the sizes of orbits in $\lfloor x\rfloor$ - which is bounded by $h(v)$. This completes the proof that (a) of the theorea implies (b).

2. To prove the positive part, we will describe the way a causal structure generates the causal net $X$ from any input net $A$ to get output net $B=f(A)$. This description will make it clear how to formally define the actual causal structure. Besides properties (i)-(iii), the only property of our functor $F$ we can use is that it is (partial) recursive. However, the way a recursive function is computed does not help us immediately to construct the causal net, because it also uses some knowledge about the individuality of the nodes of the input (we can assume that each node is a natural number), i.e. some numbering of the nodes. The causal nets, on the other hand, work in an invariant way from the beginning, without knowing about anything but the structural properties of the input. Our way to solve this difficulty (certainly not the most effective way) is to generate all possible numberings of a certain sort for the input, use them to compute the function value and then get rid of them. We need Property (iii) for the third step. Also, it will be seen that $k(P)$ for the causal structure can be made as small as the maximum of $b(G(x, A, F))$ over all inputs $A$ and output nodes $x$.

Put $N_{k}=\{1, \ldots, k\}$. Let $A$ be a connected net with $n$ elements. Any one-to-one function $u: N_{n} \mapsto A$ will be called a numbering. Let our label alphabet $\theta$ be ordered in some fixed way: $\theta=\left\{\theta_{1}, \ldots, \theta_{r}, \infty\right\}$. We also fix some pairing function $\langle i, j\rangle$ with inverse $\langle k\rangle_{1},\langle k\rangle_{2}$, with the properiy that for each $k, N_{k^{2}}=\{\langle i, j\rangle: 1 \leq i, j \leq k\}$. We order the matrices with elements from $\left\{\theta_{1}, \ldots, \theta_{r}, \infty\right\}$ lexicographically: $X=\left(x_{i j}\right)<Y=$ $\left(y_{i j}\right)$ if the sequence $\left\{x_{\langle k\rangle_{1}\langle k\rangle_{2}}: 1 \leq k \leq n^{2}\right\}$ is lexicographically smaller than $\left\{y_{\langle k\rangle_{2}\langle k\rangle_{2}}: 1 \leq k \leq n^{2}\right\}$. Each numbering $u$ of an n-node net $A$ orders to $A$ a matrix $\theta(u(i), u(j))$. This is the matrix of the net $A_{u}$ over $N_{n}$ in which the connection of $i$ and $j$ is the same as of $u(i)$ and $u(j)$ in $A$. The numberings for which the corresponding matrix is lexicographically smallest will be called frames. The net $A^{*}=A_{u}$ will be the same for each frame $u$. By restricting ourselves to frames we can reduce the set, of numberings that we have to consider. Frames can be considered as coordinate systems: a transition to a different frame is alvays accompanied by an automorphism oi $A$. Let nanely $\sigma$ be a permutation of $i_{n}, u$ a frame. $u \sigma$ is a frame again if and only if the transformation $u(i) \mapsto u(\sigma i)$ 
of $A$ is an automorphism. Thereiore fixing any frame $u$ will establish an isomorphism $\phi=u \circ \sigma \circ u^{-1}$ between the automorphism group $G$ of $A$ and the group $G^{*}$ of permutations carrying frames into frames (the dual group). We can supposa w.l.o.g. that $A=A^{*}$ (remember that on one hand, our nodes are numbers, on the other hand, a causal structure does not use these numbers anyway). In this case, the identical maping is a frame and $G=G^{*}$.

Let now $A$ be some net with possibly mora than $n$ nodes. An 2 -f fame of $A$ is a frama for sume connected subnet $C$ of $A$ with $n$ nodes. Lst $\%$ be an $n$-frame. For any $k<n$, we dencte by $u \mid k$ the restriction of the function $u: N_{n} \mapsto A$ to $N_{k}$. It is easy to see that if $u$ is an $n$-frame then $u \mid k$ is a $k$-frame for each $k<n$. This is due to our special lexicographical ordering of the matrices: if a matrix is minimal then so are all its upper left corner submatrices. We define now a sequence of nets

$$
A=C_{0} \subseteq C_{1} \subseteq \cdots
$$

representing the $k$-frames for each $k \leq n$. Suppose that $C_{n-1}$ is defined. To get $C_{n}$ we add a new node $\bar{u}$ for each $r$-frame $u$, together with two new edges: an $\alpha$-edge from $u(n)$ to $\bar{u}$ and a $\beta$-edge from $\overline{u \mid n-1}$ to $\bar{u}$ where $\alpha, \beta$ are labels not used for other purposes.

Remark 1 Suppose that some encoding $E(\sigma)$ of permutations $\sigma$ of $N_{n}$ by nets is given. An appropriate program will be able to do the following. Whenever a code $E(\sigma)$ of some permutation is brought into a certain connection with a node $\bar{u}$ of $C_{n}$ representing an $n$-frame, a new node $v$ will be generated and connected by some new edges (labelled by two new symbols used only for this purpose) to $\bar{u}$ and $\overline{u \sigma}$. In other words, it is possible to go from $\bar{u}$ to $\overline{u \sigma}$ effectively. Moreover, it is possible to do this sinultaneously for all $n$-frames $u$.

The subnet of nodes of $B$ with $n$ or less ancestors will be called the $n$-th floor $B_{n}$ of $B$. The construction of the output proceeds in many stages. In stage $n$, the $n$-ith floor will be constructed.

Let $X_{n}$ be the part of the causal net built up through the $n$-th stage. It vill contain the following parts (besides, possibly, many auxiliary nodes, from which these are distinguishable).

The input $A$.

The first $n$ floors of the output $B$.

The net $C_{n}$.

Our objective is to find a causal structure constructing $X_{n}$ from $X_{n-1}$. The first step is to construct $C_{n}$ from $C_{n-1}$ which does not present any difficulties. $B_{n}-B_{n-1}$ consists of all rodes which have exactly $n$ ancestors. For an $n$-element subset $L$ of $A$, let $B(L)$ be the set of all nodes 
of $B$ whose set of ancestors is $L$. Then $B_{n}-B_{n-1}=\cup_{L} B(L)$ and this union is disjoint. If $L_{1} \neq L_{2}$ then no edge goes between $B\left(L_{1}\right)$ and $B\left(L_{2}\right)$, otherwise the upper node on the edge would have more than $n$ aucestors. Therefore for a given $n$, all $B(L)$-s can be generated indepondently from each othar. In this way we reduced the problem to the case where

$$
\# A=n, \quad B=B_{n}, \quad B_{n}-B_{n-1}=B(A) \text {. }
$$

This case is considered further. We also have already the structure $C_{n}$ of frames of $A$.

The group $G^{F}=\left\{\sigma^{F}: \sigma \in G\right\}$ is some group of automorphisms of $B$. Two nodes $x$ and $y$ of $B$ will be called equivalent if for some $\sigma \in G^{F}$ we have $\sigma x=y$. The equivalence classes are called orbits in $B$. Let $U$ and $V$ be two orbits. We will write $U<V$ if for some $x \in U, y \in V$ we have $x<y$.

LEMMA < is a strict partial ordering of the orbits and (hence) the orbits are independent sets of nodes.

Proof: First we show that $U<V$ and $V<W$ implies $U<W$. Let $x, y_{0}, y_{1}, z$ be elements of $U, V, V, W$ respectively with $x<y_{0}, y_{1}<z$ and $\sigma y_{0}=y_{1}$. Then $\sigma x<\sigma y_{0}=y_{1}<z$ which proves $U<V$. Now we show that $U<U$ does not hold for any orbit $U$. Indeed: if $x<\sigma x$ would hold then for some $i$ we would have in our acyclic graph a cycle $x<\sigma x<\sigma^{2} x<\cdots<\sigma^{i} x=x$. $\quad \mathrm{o}$

Remark $2 B_{n}-B_{n-1}$ consist, of course, of whole orbits.

Now we introduce an invariant numbering for the orbits of $B$. Remember that we supposed that $A=A^{*}$. The nodes of $B$ are natural numbers therefore the or bits of $B$ can be lexicographicaliy ordered as sets of natural numbers. Let us use this order together with the partial order $U<V$ defined above to generate a complete order $B_{n 1}, \ldots, B_{n p}$ of orbits of $B(A)$ for which if $B_{n i}<B_{n j}$ then $i<j$. In constructing $B(A)$, the orbits $B_{n k}$ will be constructed one-by-one: we construct a sequence of nets

$$
X_{n-1}=X_{n 0} \subseteq \cdots \subseteq X_{n p}=X_{n}
$$

where $B_{n k} \subseteq X_{n k}$. Suppose that $X_{n k-1}$ has already been constructed. Our goal is to construct the nodes of $D=B_{n k}$ and to connect them to the previously constructed nodes of $B$ as required in $B_{n}$.

Let $b$ be the node of $D$ that is the smallest as a number. Let $H$ be the group of permutations $h$ of $A$ with $h^{F} b=b$. We call two frames $u$ and $v$ equivalent if $v=u h$ with some $h \in H$. The equivalence classes $u H$ 
thus defined are our candidates for the elements of $D$ : we will construct single nodes $\overline{u H}$ to represent them. The node $\overline{u H}$ will represent the node $u^{F} b$ : for arly previously constructed element $y$ of $B$, the connection of $y$ and $\overline{u H}$ will be the same as that of $y$ and $u^{F} b$ in $B$.

Now we show how to connect $a l !$ nodes $u h$ to all previously constructed nodes $y$ of $B$ with an edge expected between $y$ and $u^{F} b$. An agent sitting at node $\bar{\imath}$ has an own view of the net constructed until now. From his point of viaw, the node $y$ is represented by the number $\left(u^{-1}\right)^{F} y$. He connects it therefore to a new version of $\bar{u}$ in the way it should be connected to $b$ in $B$. This is the same connection as between $y$ and $u^{F} b$. Thus we created a new net $Y_{n k-1}$ that essentially looks like $X_{n k-1}$ except that a new copy of every node $\bar{u}$ has been created (we denote the new copy by the same symbol) with the connections to the previous parts of the net that $\overline{u H}$ should have. It remains to "merge" the nodes in $\{\overline{u h}: h \in H\}$ into a single node $\overline{u H}$ for each class $u H$.

Now we must use condition (ii). It says that for some number $k$ that is constant for our function $f$ that we want to compute, the group $H$ has a $k$-bounded chain

$$
e=G_{1} \subseteq \cdots \subseteq G_{r}=H
$$

where $\left|G_{i+1}: G_{i}\right| \leq k$. Let $G_{1}, \ldots, G_{r}$ be the first (in some lexicographical order) among those chains of $H$ with the smallest possible bound. We will construct a sequence of nets

$$
Y_{n k-1}=D_{1} \subseteq \cdots \subseteq D_{r}=X_{n k} .
$$

$D_{i}$ will contain, besides $D_{i-1}$ and some auxiliary nodes, a node $\overline{u G_{i}}$ for each equivalence class $u G_{i}$ which has the same connections to nodes in $B_{n k-1}$ as $\bar{n}$ in $Y_{n k-1}$. We also hare an edga with some special labe! from each node $\overline{u G_{i-1}}$ to $\overline{u G_{i}}$. Suppose that $D_{i-1}$ has already been constructed.

Two nodes $\overline{u G_{i-1}}$ and $\overline{v G_{i-1}}$ are considered equivalent if $v=u h$ for some $h \in G_{i}$. An equivalence class will be of the form $\left\{\overline{u h G_{i-1}}: h \in G_{i}\right\}$. Thus the elements in an equivalence class will correspond to the cosets of $G_{i-1}$ in $G_{i}$. Let $e=h_{1}, \ldots, h_{k_{i}}$ be some canonical representatives of these cosets (e.g. let each be the least in its coset in a lexicographical order of the permutations).

To construct $D_{i}$, we build up a sequence

$$
D_{i-1}=D_{i}(1) \subseteq \cdots \subseteq D_{i}\left(k_{i}\right)
$$

uf neis. $D_{i}(j)$ contains, in addition to $D_{i-1}$, for each permutation $h_{p}$ $(p \leq j)$ and each class $u G_{i-1}$ a now node $z$ which is connected by edges 
to $\overline{u G_{i-1}}$ and $\overline{u h_{p} G_{i-1}}$. These edges are labelled by a symbol $\lambda$ used only for this purpose. The node $z$ together with the two $\lambda$-edges will be called a $\lambda$-connection. If we have $D_{i}\left(k_{i}\right)$ the construction of $D_{i}$ takes only one step: each set of nodes $\left\{\overline{u h G_{i-1}}: h \in G_{i}\right\}$, together with their causes and the noles added to get $D_{i}\left(k_{i}\right)$ will form the cause of one new node, $\overline{u G_{i}}$. In this same step, this new node can be made to have the same connections is $B_{n-1-1}$ as $\overline{u G_{i-1}}$.

Our only remaining task is thesefore to construct $D_{i}(j)$ from $D_{i}(j-1)$. This will bappen through a sequence of nets

$$
D_{i}(j-1) \subseteq E_{1} \subseteq \cdots \subseteq E_{i-1}=D_{i}(j)
$$

$E_{m}$ will have, in addition to $D_{i}(j-1)$, a $\lambda$-connection between each node $\overline{u G_{i-1}}$ and $\overline{u h_{j} G_{m}}$. To construct $E_{1}$ from $D_{i}(j-1)$ we must first construct for each frame $u$ a new node representing $u$ (we denote it also by $\bar{u}$ ) together with a $\lambda$-connection from node $\overline{u G_{i-1}}$ to this new node. Then we make a $\lambda$-connection from the node $\bar{u}$ to $\overline{u h_{j}}$ using Remark 1 . This connection can be used to generate a $\lambda$-connection between $\overline{u G_{i-1}}$ and $\overline{u h_{j}}$. It is easy to see that the construction of $E_{m}$ from $E_{m-1}$ will take only one step for each $m$.

\section{A recursive function with no recursive functor}

The domain of definition of our function $f$ will be the set of certain 0-1 sequences. There is an obvious encoding of these sequences into nets. We will suppose that e.g. the sequence 01 and 10 are isomorphic (reversal is an isomorphism). $f$ is defined over all $\left\{a_{n k}=101^{n} 0^{k}: n, k>0\right\}$ and $\left\{b_{n k}=1101^{n} 0^{k}: n, k>0\right\}$. We will use the $\mu$-operator from recursion theory. If for some function $g, g(k)=0$ for all $k<n$ then we put $\mu_{k<n} g(k)=n$. Let $g(n, k)$ be a number-theoretical function for which the predicate $P(n) \Leftrightarrow g\left(n, \mu_{k} g(n, k) \neq 0\right)=1$ is undecidable. Put $G(n, k)=$ $g\left(n, \mu_{j<k} g(n, k) \neq 0\right)$. Put

$$
\begin{aligned}
& f\left(a_{n k}\right)= \begin{cases}001 & \text { if } G(n, k)=0 \\
0001 & \text { otherwise. }\end{cases} \\
& f\left(b_{n k}\right)= \begin{cases}c=00101100 & \text { if } G(n, k)=0 \\
0 c & \text { if } G(n, k)=1 \\
c 0 & \text { otherwise. }\end{cases}
\end{aligned}
$$

Obviously, the only embeddings in this domain are the unique embeddings of $a_{n k}$ to $a_{n k+1}, b_{n k}$ to $b_{n k+1}, a_{n k}$ to $b_{n k}$ and tha combinations of these. 
The functor $F$ must correspond an embedding from $f\left(a_{n k}\right)$ to $f\left(b_{n k}\right)$ to the last type mentioned. If $G(n, k)=0$, the functor has two possible values: ye can embed 001 to the front or the end or $c$. But if $G(n, k) \neq 0$, there is only one embedding: either to the front or to the back. Notice that $a_{x 21}$ can also be embedcled to $a_{n k}$. Therefore, the functor property implies that the embedding of $f\left(a_{n k}\right)$ to $f\left(b_{n k}\right)$ must be a continuation of the conedding of $f\left(a_{n 1}\right)$ to $f\left(j_{n 1}\right)$, and $f\left(a_{n 1}\right)$ will be crnbeddad to the fiont as back of $f\left(b_{n 1}\right)$ deperiding on $P(n)$, i.e. in a nonrecursive way. 


\section{REFERENCES}

[1] A. N. Kolmogoroy, V. A. Uspenskii: On the Definition of an Algorithm, Uspehi Mat. Nauk 13(1958) 3-28; AMS Transl. 2nd ser. 29 (1933) 217-245.

[2] N. V. Petri: Personal communication.

[3] A. Schönhage: Storage Modification Machines, SIAM J. on Compubing 9/3 (Aug. 1980) 49C-508.

[4] Ja. M. Barzdin', Ja. Ja. Kainin's: A Universal Automaton with Variable Structure, Autcratic Control and Computing Sciences 6 (1974/2)

[5] L. Babai, L. Lovász: Permutation Groups and Almost Regular Graphs, Studia Sci. Math. Hung. 8 (1973) 141-150.

[6] S. A. Cook: An Observation on Time-Storage Trade-Off, Proc. Fifth Ann. ACM Symp. on the Theory of Computing, (1973) 29-33.

[7] E. M. Luks: Isomorphism of Graphs of Bounded Valence Can Be Tested in Polynomial Time, Proc. of the $21^{\text {th }}$ Symp. on FOCS, Syracuse 1980. 